\title{
A Direct Evaluation of the Partition Function and Thermodynamic Data for Water at High Temperatures
}

\author{
Frederico V. Prudente ${ }^{\dagger}$ and António J. C. Varandas* \\ Departamento de Química, Universidade de Coimbra, 3004-535 Coimbra, Portugal
}

Received: March 25, 2002; In Final Form: May 1, 2002

\begin{abstract}
The rovibrational partition function of the water molecule is calculated using a classical statistical mechanics approach and a hybrid method recently proposed by Prudente et al. [J. Phys. Chem. A 2001, 105, 5272], which corrects the classical results. The phase-space integrals are solved using a Monte Carlo technique. For temperatures between 500 and $6000 \mathrm{~K}$, the results are compared with previous approximate and exact quantum calculations. Estimates of some thermodynamic quantities for gas-phase water as a function of temperature are also reported and compared with previous results. The calculated partition function, Gibbs enthalpy, Helmholtz function, entropy, and specific heat at constant pressure indicate that the hybrid scheme can provide accurate thermodynamic data for polyatomic molecules at high temperatures.
\end{abstract}

\section{Introduction}

Accurate thermodynamic data of gas-phase polyatomic systems are of great importance in chemistry and physics. In principle, the partition function, and hence other thermodynamic properties, can be evaluated exactly in quantum statistical mechanics by summing directly over all of the energy levels of the system (so-called sum-over-states). Although an enormous advance has been made in recent years along this line of approach, the calculation of rovibrational states is currently feasible only for systems with a few degrees of freedom, ${ }^{1-5}$ which limits the applicability of the sum-over-states approach to small molecules. ${ }^{6-9}$ To overcome this problem, the partition function and related thermodynamic data have been traditionally calculated by fitting effective Hamiltonians to experimental data. ${ }^{10-13}$ However, the accuracy of the results obtained by using the traditional methods is expected to be poor at high temperatures $^{8,14}$ and for floppy (anharmonic) systems. ${ }^{15}$

For the above reasons, several procedures have been proposed as routes to the direct sum-over-states approach and fitting of experimental data. These include the hybrid analytic/direct summation method of ab initio calculations, ${ }^{14}$ Fourier pathintegral Monte Carlo methods, ${ }^{16-18}$ and classical statistical mechanics (CSM) methods both with consideration of quantum, ${ }^{19}$ semiclassical, ${ }^{20}$ and semiempirical ${ }^{21}$ corrections and without consideration of such corrections. ${ }^{15,22-25}$ In a previous paper, ${ }^{26}$ we surveyed briefly the most popular classical methods, which employ corrections of various types, and proposed a novel scheme (hybrid LCP/QFH), which consists of adding an effective potential to the classical Hamiltonian to mimic quantum effects. Such a method blends the advantages of the linear classical path ${ }^{27}$ (LCP) and the quadratic FeynmanHibbs $^{28}$ (QFH) methods while avoiding their nondesirable features. In fact, preliminary calculations for diatomic molecules ${ }^{26}$ have shown that the hybrid LCP/QFH method performs generally better than previous approaches for moderate and high temperatures.

\footnotetext{
* To whom correspondence should be addressed. E-mail address: varandas@qtvs1.qui.uc.pt.

$\dagger$ Present address: Instituto de Føsica, Universidade Federal da Bahia, 40210-340 Salvador, BA, Brazil. E-mail: prudente@ufba.br
}

A major goal of this work is to extend the hybrid LCP/QFH calculations of the rovibrational partition function and thermodynamic properties [e.g, the Gibbs enthalpy function (gef), Helmholtz function (hcf), entropy $(S)$, and specific heat capacity at constant pressure $\left(C_{p}\right)$ ] of gas-phase triatomic systems described by realistic potential energy surfaces. Thus, we envisage a simple and relatively inexpensive computational scheme amenable to generalization to multidimensional systems and that can provide accurate internal partition functions (and other thermodynamic data) for such polyatomic systems. Conversely to previous work, ${ }^{15,23,26,29}$ the multidimensional phase-space integrals that appear in the classical formalism will be evaluated by using a crude Monte Carlo method to sample the coordinates, while the Barker Monte Carlo algorithm ${ }^{30}$ will be used to sample the conjugate momenta (see later).

As a case study, we consider the $\mathrm{H}_{2} \mathrm{O}$ molecule in its ground electronic state. Indeed, water is the most common polyatomic molecule in the universe, being fundamental to life and an essential constituent of the Earth's atmosphere. Its thermodynamic data at high temperatures is therefore of great importance for modeling combustion, exhaust gases, and the atmosphere of cool stars, just to mention a few examples. Moreover, the $\mathrm{H}_{2} \mathrm{O}$ molecule is representative of molecular systems with a deep potential well and commonly plays the role of a benchmark system both for bound-state and for reactive scattering calculations $\left[\mathrm{O}\left({ }^{1} \mathrm{D}\right)+\mathrm{H}_{2}\right.$ reaction]. It is also known for the poor results of the partition functions that are obtained even at moderate and high temperatures when using CSM. ${ }^{20,23}$ Because of relatively simple and fundamental characteristics of water, there are many predictions of its partition function and thermodynamic properties, ${ }^{7,8,10-14,18,20,23,31}$ including the more recent and accurate estimation obtained by Vidler and Tennyson. ${ }^{32}$ This will allow a detailed test of our method.

The paper is organized as follows. In section 2, we discuss the calculation of the internal partition function and related thermodynamic properties by using the standard classical statistical mechanics and hybrid LCP/QFH methods and summarize the Monte Carlo procedure utilized to evaluate the involved multidimensional phase-space integrals. The details of the calculations and results are presented in section 3, while some conclusions are in section 4. 


\section{Methodology}

2.1. General. The molecular partition function is usually expressed as

$$
Q=Q_{\text {tr }} Q_{\text {elec }} Q_{\text {rovib }}
$$

where $Q_{\mathrm{tr}}, Q_{\text {elec }}$, and $Q_{\text {rovib }}$ are the translational, electronic, and rovibrational contributions, respectively. Although $Q_{\text {tr }}$ can be calculated using the ideal gas formalism ${ }^{33}$ and $Q_{\text {elec }}$ can be assumed to be unity (because no electronic excited states are involved; $;^{8,14}$ for a discussion on this issue, see ref 34 ), $Q_{\text {rovib }}$ has to be evaluated from the potential energy surface by using quantum statistical mechanics or an approximate procedure. In this work, we use two formulations based on classical statistical mechanics. In the first approach, $Q_{\text {rovib }}$ assumes the standard classical form ${ }^{33,35}$

$$
Q_{\text {rovib }}^{\mathrm{CM}}(T)=\frac{\exp \left(\beta \epsilon_{0}\right)}{h^{n}} \iint_{\mathscr{S}} \exp \left[-\beta H^{\mathrm{CM}}(\mathbf{q}, \mathbf{p})\right] \mathrm{d} \mathbf{q} \mathrm{d} \mathbf{p}
$$

where $H^{\mathrm{CM}}(\mathbf{q}, \mathbf{p})$ is the classical Hamiltonian, $\epsilon_{0}$ is the zeropoint energy of the system, $\beta=1 /\left(k_{\mathrm{B}} T\right), k_{\mathrm{B}}$ is the Boltzmann constant, $T$ is the temperature, $h$ is the Planck constant, $n$ is the number of degrees of freedom, $\mathbf{q}$ is the generalized coordinate vector, and $\mathbf{p}$ is the corresponding conjugate momenta. In the second approach, we employ the hybrid LCP/QFH method, which corrects the classical rovibrational partition function by adding an effective potential to the classical Hamiltonian. One has $^{26}$

$$
\begin{array}{r}
Q_{\text {rovib }}^{\mathrm{LCP} / \mathrm{QFH}}(T)=\frac{\exp \left(\beta \epsilon_{0}\right)}{h^{n}} \iint_{\mathscr{S}} \exp \left\{-\beta\left[H^{\mathrm{CM}}(\mathbf{q}, \mathbf{p})+\right.\right. \\
\left.\left.V^{\mathrm{eff}}(\mathbf{q})\right]\right\} \mathrm{dq} \mathrm{d} \mathbf{p}
\end{array}
$$

where the effective potential is given by

$$
V^{\mathrm{eff}}(\mathbf{q})=\beta A \nabla^{2} V(\mathbf{q})+\beta^{2} A(\nabla \cdot V(\mathbf{q}))^{2}
$$

with $A=\hbar^{2} /(48 \mu)$. The first term $(\times 2)$ of eq 4 is the quadratic Feynman-Hibbs (QFH) approximation ${ }^{28}$ of the Feynman path integral formulation, while the second one $(\times 2)$ is the linear classical approximation (LCP) due to Miller. ${ }^{27}$ We have shown in a previous paper ${ }^{26}$ that the QFH method generally underestimates the values of the quantum partition function, while the LCP approximation overestimates the values at low and moderate temperatures. Note that the subscript $\mathscr{B}$ in eqs 2 and 3 implies that the hypervolume of integration is restricted to phase-space regions corresponding to a bound-state situation: ${ }^{36} 0 \leq H^{\mathrm{CM}}(\mathbf{q}, \mathbf{p}) \leq D_{\mathrm{e}}$, where $D_{\mathrm{e}}$ is the classical dissociation energy of the molecule with the minimum of the potential energy surface assumed as the reference energy. In turn, the factor exp$\left(\beta \epsilon_{0}\right)$ in eqs 2 and 3 is required to compare with previous results for water, which have been calculated by assuming the zeropoint energy $\left(\epsilon_{0}\right)$ as the reference energy.

A temperature-dependent estimate of the thermodynamic quantities considered in this work can be obtained from the partition function and its first and second moments, the rovibrational contributions of which are defined by ${ }^{14}$

$$
\begin{gathered}
Q_{\text {rovib }}^{\prime}=T \frac{\mathrm{d} Q_{\text {rovib }}}{\mathrm{d} T} \\
Q_{\text {rovib }}^{\prime \prime}=T^{2} \frac{\mathrm{d}^{2} Q_{\text {rovib }}}{\mathrm{d} T^{2}}+2 Q_{\text {rovib }}^{\prime}
\end{gathered}
$$

where the rovibrational partition function is expressed as in eqs 2 or 3 . In principle, the moments could be obtained through numerical differentiation of $Q_{\mathrm{rovib}}^{\mathrm{CM}}$ or $Q_{\mathrm{rovib}}^{\mathrm{LCP} / \mathrm{QFH}}$, although it is more advantageous to differentiate them analytically. For the standard classical approach, the resulting expressions are

$$
\begin{gathered}
Q_{\text {rovib }}^{\prime \mathrm{CM}}=\frac{1}{h^{n}} \iint_{S} \beta \tilde{H}^{\mathrm{CM}}(\mathbf{q}, \mathbf{p}) \exp \left[-\beta \tilde{H}^{\mathrm{CM}}(\mathbf{q}, \mathbf{p})\right] \mathrm{d} \mathbf{q} \mathrm{d} \mathbf{p} \\
Q_{\text {rovib }}^{\prime \prime \mathrm{CM}}=\frac{1}{h^{n}} \iint_{\mathscr{S}}\left[\beta \tilde{H}^{\mathrm{CM}}(\mathbf{q}, \mathbf{p})\right]^{2} \exp \left[-\beta \tilde{H}^{\mathrm{CM}}(\mathbf{q}, \mathbf{p})\right] \mathrm{d} \mathbf{q} \mathrm{d} \mathbf{p}
\end{gathered}
$$

where $\tilde{H}^{\mathrm{CM}}(\mathbf{q}, \mathbf{p})=H^{\mathrm{CM}}(\mathbf{q}, \mathbf{p})-\epsilon_{0}$, while for the hybrid LCP/ $\mathrm{QFH}$ approach, one has

$$
\begin{aligned}
& Q_{\text {rovib }}^{\prime \mathrm{LCP} / \mathrm{QFH}}=\frac{1}{h^{n}} \iint_{\mathscr{B}}\left[\beta \tilde{H}^{\mathrm{CM}}(\mathbf{q}, \mathbf{p})+2 \beta^{2} A \nabla^{2} V(\mathbf{q})+\right. \\
& \left.3 \beta^{3} A(\nabla \cdot V(\mathbf{q}))^{2}\right] \exp \left\{-\beta\left[\tilde{H}^{\mathrm{CM}}(\mathbf{q}, \mathbf{p})+V^{\mathrm{eff}}(\mathbf{q})\right]\right\} \mathrm{dq} \mathrm{d} \mathbf{p}(9) \\
& Q_{\text {rovib }}^{\prime \prime \mathrm{LCP} / \mathrm{QFH}}=\frac{1}{h^{n}} \iint_{\mathscr{B}}\left\{\left[\beta \tilde{H}^{\mathrm{CM}}(\mathbf{q}, \mathbf{p})+2 \beta^{2} A \nabla^{2} V(\mathbf{q})+\right.\right. \\
& \left.\left.3 \beta^{3} A(\nabla \cdot V(\mathbf{q}))^{2}\right]^{2}-2 \beta^{2} A \nabla^{2} V(\mathbf{q})-6 \beta^{3} A(\nabla \cdot V(\mathbf{q}))^{2}\right\} \\
& \quad \exp \left\{-\beta\left[\tilde{H}^{\mathrm{CM}}(\mathbf{q}, \mathbf{p})+V^{\mathrm{eff}}(\mathbf{q})\right]\right\} \mathrm{dq} \mathrm{d} \mathbf{p}(10)
\end{aligned}
$$

The ideal gas thermodynamic functions as a function of temperature can be obtained in terms of $Q_{\text {rovib }}, Q_{\text {rovib }}^{\prime}$ and $Q_{\text {rovib }}^{\prime \prime}$ as follows:

\section{The Gibbs enthalpy function}

$$
\operatorname{gef}(T)=-\frac{\left[G(T)-H_{0}\right]}{T}=R \ln Q_{\text {rovib }}+\operatorname{gef}^{\mathrm{tr}}(T)+\frac{H_{0}}{T}
$$

\section{The Helmholtz function}

$$
\operatorname{hcf}(T)=H(T)-H_{0}=R T \frac{Q_{\text {rovib }}^{\prime}}{Q_{\text {rovib }}}+\operatorname{hcf}^{\mathrm{tr}}(T)-H_{0}
$$

\section{The entropy}

$$
S(T)=R \frac{Q_{\text {rovib }}^{\prime}}{Q_{\text {rovib }}}+R \ln Q_{\text {rovib }}+S^{\mathrm{tr}}(T)
$$

\section{The specific heat capacity at constant pressure}

$$
C_{p}(T)=R\left[\frac{Q_{\text {rovib }}^{\prime \prime}}{Q_{\text {rovib }}}-\left(\frac{Q_{\text {rovib }}^{\prime}}{Q_{\text {rovib }}}\right)^{2}\right]+C_{p}^{\mathrm{tr}}
$$

where $\operatorname{gef}^{\mathrm{tr}}(T), \operatorname{hcf} f^{\operatorname{tr}}(T), S^{\operatorname{tr}}(T)$, and $C_{p}^{\mathrm{tr}}(T)$ are the translational contributions, which, for an ideal gas, assume the form ${ }^{10}$

$$
\begin{gathered}
\operatorname{gef}^{\mathrm{tr}}(T)=R\left[\frac{3}{2} \log M+\frac{5}{2} \log T+\log \left(\frac{k_{\mathrm{B}}}{p}\left(\frac{2 \pi k_{\mathrm{B}}}{h^{2}}\right)^{3 / 2}\right)\right] \\
\operatorname{hcf}^{\mathrm{tr}}(T)=\frac{5}{2} R T \\
S^{\mathrm{tr}}(T)=\operatorname{gef}^{\mathrm{tr}}(T)+\frac{5}{2} R \\
C_{p}^{\mathrm{tr}}(T)=\frac{5}{2} R
\end{gathered}
$$

with $R$ being the gas constant and $p$ the pressure. The $H_{0}$ constant, which appears in $\operatorname{gef}(T)$ and $\operatorname{hcf}(T)$, is the reference enthalpy at the JANAF reference temperature of $298.15 \mathrm{~K},{ }^{10}$ 
that is, $H(298.15)$. Because the results obtained within the classical framework have poor accuracy at low temperatures, we will utilize in our calculations the value of $H_{0}=9904.1 \mathrm{~J}$ $\mathrm{mol}^{-1}$ derived by Vidler and Tennyson ${ }^{32}$ from both theoretical and experimental energy levels. Such a value is close to the one reported in the JANAF tables $\left(H_{0}=9904 \mathrm{~J} \mathrm{~mol}^{-1}\right)$ and can be compared with the calculated values of Martin et al. ${ }^{14}$ and Harris et al. ${ }^{8}$ (respectively, $H_{0}=9902 \mathrm{~J} \mathrm{~mol}^{-1}$ and $H_{0}=$ $9895.4 \mathrm{~J} \mathrm{~mol}^{-1}$ ). Although arbitrary, such a choice leads to errors smaller in magnitude than the statistical errors inherent to our Monte Carlo calculations, even at low temperatures.

2.2. Classical Hamiltonian and Effective Potential. The next step consists of obtaining the expressions for the classical Hamiltonian $\left(H^{\mathrm{CM}}\right)$ and the effective potential $\left(V^{\text {eff }}\right)$ used to calculate the rovibrational partition function of a system with three structureless particles; $m_{i}$ will be the mass of the $i$-th particle, and $\mathbf{X}_{i}$ will be its position vector with respect to the space-fixed axes. The rovibrational motion of the three particles relative to the center of mass of the system can be described by using mass-weighted Jacobi vectors ${ }^{37}$

$$
\begin{gathered}
\mathbf{r}=d^{-1}\left(\mathbf{X}_{3}-\mathbf{X}_{2}\right) \\
\mathbf{R}=d\left(\mathbf{X}_{1}-\frac{m_{2} \mathbf{X}_{2}+m_{3} \mathbf{X}_{3}}{m_{2}+m_{3}}\right)
\end{gathered}
$$

and their corresponding conjugate momenta $\mathbf{P}_{\mathbf{r}}$ and $\mathbf{P}_{\mathbf{R}}$, which defines a 12-dimensional (12D) phase space. In eqs 19 and 20, $d$ is the mass scaling or normalizing factor,

$$
d=\left[\left(\frac{m_{1}}{\mu}\right)\left(1-\frac{m_{1}}{M}\right)\right]^{1 / 2}
$$

and

$$
\mu=\left(\frac{m_{1} m_{2} m_{3}}{M}\right)^{1 / 2}
$$

is the three-body reduced mass; $M=m_{1}+m_{2}+m_{3}$ is the total mass of the system. With the use of this phase-space coordinates set, the expression of the classical Hamiltonian assumes the form

$$
\begin{aligned}
H\left(\mathbf{R}, \mathbf{r}, \mathbf{P}_{\mathbf{R}}, \mathbf{P}_{\mathbf{r}}\right) & =\frac{\mathbf{P}_{\mathbf{R}}{ }^{2}}{2 \mu}+\frac{\mathbf{P}_{\mathbf{r}}{ }^{2}}{2 \mu}+V(\mathbf{R}, \mathbf{r}) \\
& =\frac{P_{\mathbf{R}}{ }^{2}}{2 \mu}+\frac{P_{\mathbf{r}}{ }^{2}}{2 \mu}+V(R, r, \theta)
\end{aligned}
$$

where $R=|\mathbf{R}|, r=|\mathbf{r}|$, and $\theta=\cos ^{-1}(\mathbf{R} \cdot \mathbf{r} /(R r))$ are the internal mass-weighted Jacobi coordinates, $P_{\mathbf{R}}=\left|\mathbf{P}_{\mathbf{R}}\right|$, and $P_{\mathbf{r}}=\left|\mathbf{P}_{\mathbf{r}}\right|$. Note that the classical Hamiltonian depends explicitly only on the variables $R, r, \theta, P_{\mathbf{R}}$ and $P_{\mathbf{r}}$. Note further that the interatomic distances $\left(r_{12}, r_{13}, r_{23}\right)$ and internal mass-weighted Jacobi coordinates are related by

$$
\begin{gathered}
r_{23}=d r \\
r_{13}{ }^{2}=\left(\frac{m_{2} d r}{m_{2}+m_{3}}\right)^{2}+\frac{R^{2}}{d^{2}}-\frac{2 m_{2}}{m_{2}+m_{3}} r R \cos \theta \\
r_{12}{ }^{2}=\left(\frac{m_{3} d r}{m_{2}+m_{3}}\right)^{2}+\frac{R^{2}}{d^{2}}+\frac{2 m_{3}}{m_{2}+m_{3}} r R \cos \theta
\end{gathered}
$$

Thus, the phase-space integrals, which appear in the classical rovibrational partition function (without any correction) and the hybrid LCP/QFH method, as well as on the corresponding moments, can be written in terms of internal mass-weighted Jacobi coordinates and the moduli of the conjugate momenta as follows:

$$
\begin{aligned}
I= & \frac{1}{h^{6}} \iint_{\mathscr{B}} F\left(\mathbf{R}, \mathbf{r}, \mathbf{P}_{\mathbf{R}}, \mathbf{P}_{\mathbf{r}}\right) \mathrm{d} \mathbf{R} \mathrm{d} \mathbf{r} \mathrm{d} \mathbf{P}_{\mathbf{R}} \mathrm{d} \mathbf{P}_{\mathbf{r}} \\
= & \frac{128 \pi^{4}}{h^{6}} \iint_{\mathscr{B}} F\left(R, r, \theta, P_{\mathbf{R}}, P_{\mathbf{r}}\right) R^{2} r^{2} P_{\mathbf{R}}^{2} P_{\mathbf{r}}^{2} \mathrm{~d} \mathbf{R} \mathrm{d} \mathbf{r} \mathrm{d} \\
& (\cos \theta) \mathrm{d} P_{\mathbf{R}} \mathrm{d} P_{\mathbf{r}}
\end{aligned}
$$

Such expressions can be obtained by adopting spherical polar coordinates to describe $\mathbf{R}, \mathbf{r}, \mathbf{P}$, and $\mathbf{p}$ and performing analytically all of the integrals involving coordinates on which the Hamiltonian does not depend explicitly. Note that the multidimensional phase-space integral is then reduced from 12D to 5D. The function $F(\cdots)$ collects the integrands of eqs $2,3,7,8$, 9 , and 10 .

Moreover, for the hybrid LCP/QFH method, one requires the effective potential [eq 4] in terms of internal mass-weighed Jacobi coordinates. After some simple algebra, we can write the terms in $\beta$ and $\beta^{2}$ as

$$
\begin{aligned}
& \nabla^{2} V= \nabla_{\mathbf{R}}^{2} V+\nabla_{\mathbf{r}}^{2} V \\
&=\frac{\partial^{2} V}{\partial R^{2}}+\frac{2}{R} \frac{\partial V}{\partial R}+\frac{\partial^{2} V}{\partial r^{2}}+\frac{2}{r} \frac{\partial V}{\partial r}+ \\
&\left(\frac{1}{R^{2}}+\frac{1}{r^{2}}\right)\left(\frac{\partial^{2} V}{\partial \theta^{2}}+\operatorname{cotg} \theta \frac{\partial V}{\partial \theta}\right)
\end{aligned}
$$

and

$$
\begin{aligned}
(\nabla V)^{2} & =\left(\nabla_{\mathbf{R}} V\right)^{2}+\left(\nabla_{\mathbf{r}} V\right)^{2} \\
& =\left(\frac{\partial V}{\partial R}\right)^{2}+\left(\frac{\partial V}{\partial r}\right)^{2}+\left(\frac{1}{R^{2}}+\frac{1}{r^{2}}\right)\left(\frac{\partial V}{\partial \theta}\right)^{2}
\end{aligned}
$$

Finally, to perform the integratations involved in the rovibrational partition function and related thermodynamic quantities, we employ a Monte Carlo procedure, which will be described next.

2.3. Monte Carlo Approach. The methods generically classified as Monte Carlo offer one of the most powerful techniques to evaluate multidimensional integrals (e.g., see refs 38 and 39). Examples of their use in chemical physics are the determination of classical partition functions and density of states for molecular systems with realistic potential energy surfaces. ${ }^{40-49}$ In previous work, ${ }^{15,23,26,29}$ we have used such a method based on an adaptation of the Monte Carlo algorithm originally reported by Barker $^{30}$ within the context of transitionstate theory. The spirit of such an algorithm is akin to the idea of importance sampling and consists of choosing a sampling domain that coincides as much as possible with the integration domain. Thus, the variables are not sampled independently of each other, but instead some kind of dependence is introduced. This leads to a normalized but nonuniform distribution and, hence, requires the use of appropriate weighting factors (see refs 29 and 30 for details).

However, as pointed out elsewhere, ${ }^{23}$ the sampling of the configurational space for systems of which the potential energy surfaces possess two or more minima is not a trivial matter when using the Barker algorithm. In fact, the sampling becomes complicated and time-consuming, which led us to utilize here 
the simpler crude Monte Carlo approach to sample the internal mass-weighed Jacobi coordinates $(R, r, \theta)$. However, we keep using Barker's algorithm to sample the modulus of the conjugate momenta $\left(P_{\mathbf{R}}\right.$ and $\left.P_{\mathbf{r}}\right)$. Note that in the crude Monte Carlo integration the variables are sampled independently of each other using a sequence of pseudorandom numbers, which generate a uniform distribution over the configuration space. As already noted, all multidimensional integrals encountered here have the general form of eq 25, and hence, we summarize below the general procedure adopted to evaluate them. It involves the following steps:

(1) Define a minimum and maximum displacement for each of the internal mass-weigthed Jacobi coordinates, namely, $R^{\mathrm{min}}$, $R^{\max }, r^{\min }, r^{\max }, \theta^{\min }$, and $\theta^{\max }$, so that the sampled hyperrectangular volume defined by these three coordinate intervals includes the true volume of integration (i.e., $V(R, r, \theta) \leq D_{\mathrm{e}}$ for $\left.P_{\mathbf{R}}=P_{\mathbf{r}}=0\right)$ but is as much as possible close to it.

(2) Sample randomly $R, r$, and $\cos (\theta)$ within their range to obtain the values $R^{\mathrm{S}}, r^{\mathrm{S}}$, and $\theta^{\mathrm{S}}$ according to

$$
\begin{gathered}
R^{\mathrm{S}}=R^{\min }+\left(R^{\max }-R^{\min }\right) \xi \\
r^{\mathrm{S}}=r^{\min }+\left(r^{\max }-r^{\min }\right) \xi \\
\cos \left(\theta^{\mathrm{S}}\right)=\cos \left(\theta^{\max }\right)+\left(\cos \left(\theta^{\min }\right)-\cos \left(\theta^{\max }\right)\right) \xi
\end{gathered}
$$

where $\xi$ is a random number in the range $[0,1]$.

(3) Calculate the potential at the sampled point, $V^{S}=$ $V\left(R^{\mathrm{S}}, r^{\mathrm{S}}, \theta^{\mathrm{S}}\right)$. If it represents a bound-state situation (i.e., $V^{\mathrm{S}} \leq$ $D_{\mathrm{e}}$ ), move to the next step. Otherwise (i.e., the sampled point lies outside the true hypervolume of integration $\mathscr{B}$ ), go to step 6.

(4) Following Barker's procedure, find the minimum and maximum displacements and the sampled value for each conjugate momenta $P_{\mathbf{R}}$ and $P_{\mathbf{r}}$ according to

$$
\begin{gathered}
P_{\mathbf{R}}^{\min }=0 \\
P_{\mathbf{R}}^{\max }=\sqrt{2 \mu\left(D_{\mathrm{e}}-V^{\mathrm{S}}\right)} \\
P_{\mathbf{R}}^{\mathrm{S}}=P_{\mathbf{R}}^{\max \xi} \\
P_{\mathbf{r}}^{\min }=0 \\
P_{\mathbf{r}}^{\max }=\sqrt{2 \mu\left(D_{\mathrm{e}}-V^{\mathrm{S}}\right)-\left(P_{\mathbf{R}}^{\mathrm{S}}\right)^{2}} \\
P_{\mathbf{r}}^{\mathrm{S}}=P_{\mathbf{r}}^{\max \xi}
\end{gathered}
$$

The sampled point $\mathbf{x}_{\mathrm{g}}^{\mathrm{S}}=\left(R^{\mathrm{S}}, r^{\mathrm{S}}, \theta^{\mathrm{S}}, P_{\mathbf{R}}^{\mathrm{S}}, P_{\mathbf{r}}^{\mathrm{S}}\right)$ is therefore within the hypervolume of integration $\mathscr{B}$.

(5) Calculate the weight factor associated with the sampled point $\mathbf{x}_{\mathrm{g}}^{\mathrm{S}}$ according to

$$
w_{\mathrm{g}}=\frac{128 \pi^{4}}{h^{6}} W_{R} W_{r} W_{P_{\mathbf{R}}} W_{P_{\mathbf{r}}}
$$

where

$$
\begin{gathered}
W_{R}=\left(R^{\mathrm{S}}\right)^{2}\left(R^{\max }-R^{\min }\right)\left(\cos \left(\theta^{\min }\right)-\cos \left(\theta^{\max }\right)\right) \\
W_{\mathrm{r}}=\left(r^{\mathrm{S}}\right)^{2}\left(r^{\max }-r^{\min }\right) \\
W_{P_{\mathbf{R}}}=\left(P_{\mathbf{R}}^{\mathrm{S}}\right)^{2} P_{\mathbf{R}}^{\max } \\
W_{\mathrm{P}_{\mathbf{r}}}=\left(P_{\mathbf{r}}^{\mathrm{S}}\right)^{2} P_{\mathbf{r}}^{\max }
\end{gathered}
$$

which represents the hypervolume (divided by $h^{6}$ ) associated with $\mathbf{x}_{\mathrm{g}}$.
(6) Repeat $N_{\mathrm{T}}$ times the steps $2-5$ to evaluate the integral of the eq 25 as

$$
I \approx I^{N_{\mathrm{T}}}=\frac{1}{N_{\mathrm{T} g=1}} \sum_{\mathrm{in}}^{N_{g}} w_{g} F_{g}
$$

where $F_{g}=F\left(\mathbf{x}_{\mathrm{g}}^{\mathrm{S}}\right) \equiv F\left(R^{\mathrm{S}}, r^{\mathrm{S}}, \theta^{\mathrm{S}}, P_{\mathbf{R}}^{\mathrm{S}}, P_{\mathbf{r}}^{\mathrm{S}}\right)$ is the function to be integrated, $N_{\mathrm{T}}$ is the total number of sampled points, and $N_{\text {in }}$ is the total number of sampled points that are within the hypervolume of integration $\mathscr{B}$. The standard deviation associated with eq 31 assumes the form

$$
\sigma^{2}=\frac{1}{N_{\text {in }}\left(N_{\text {in }}-1\right)} \sum_{g=1}^{N_{\text {in }}}\left(\frac{N_{\text {in }}}{N_{\mathrm{T}}} w_{g} F_{g}-I^{N_{\mathrm{T}}}\right)^{2}
$$

Note that the efficiency of this Monte Carlo procedure, which is defined by $\epsilon=N_{\mathrm{in}} / N_{T}$, is not close to 1 as in Barker's algorithm but is certainly larger than for the crude Monte Carlo method because Barker's method is still employed to sample the momenta. Note further that such a fact does not imply that the present Monte Carlo procedure requires a larger computational effort than the one based on Barker's algorithm. The reason is that the determination of an integration domain close to the true hypervolume $\mathscr{B}$ as required in Barker's algorithm can itself be time-consuming because of the necessity of calling the potential-energy surface routine many times.

\section{Technical Details and Results}

The rovibrational partition function and its first and second moments have been computed from the standard classical statistical mechanics expressions in eqs 2,7 , and 8 by using the Monte Carlo method described above. Calculations have also been carried out by using the hybrid LCP/QFH method (eqs 3, 9, and 10). Moreover, calculations of thermodynamic functions are reported from $Q_{\text {rovib }}, Q_{\text {rovib }}^{\prime}$, and $Q_{\text {rovib }}^{\prime \prime}$ by employing eqs $11-14$. All calculations considered a standard state pressure of $p=1$ bar $\left(10^{5} \mathrm{~Pa}\right)$ as used in the $\mathrm{JANAF}^{10}$ tables and by Vidler and Tennyson. ${ }^{32}$ To describe the $\mathrm{H}_{2} \mathrm{O}$ molecule, we have employed the energy-switching (ES) potential energy surface reported by one of us. ${ }^{50}$ This has been obtained by merging a modified form of the global many-body expansion (MBE) potential of Murrell and Carter ${ }^{51}$ and a spectroscopically accurate polynomial form reported by Polyansky et al. ${ }^{52}$ (known as PJT1). The classical dissociation energy of the ES potential energy surface is $D_{\mathrm{e}}=0.19986554 E_{\mathrm{h}}$. Besides being global and having spectroscopic accuracy where this is known, such a potential energy surface offers the advantadge of its analytical first derivatives with respect to the internuclear distances having been obtained. ${ }^{53}$ Thus, only its second derivatives are needed to be calculated numerically for the purpose of evaluating the effective potential (eq 4) according to the hybrid LCP/QFH method. They have been calculated numerically from the known analytical first-derivatives, thus avoiding errors inherent to the numerical estimation of high-order derivatives.

Before we present the results, we should define the $R, r$, and $\theta$ displacement intervals, of which the importance has been highlighted in the previous section. Thus, to improve the efficiency of the Monte Carlo procedure, we should establish the smallest hyperrectangle that contains the domain of integration $\mathscr{B}$. This can be defined by the minimum values of $R$ and $r$ when varied independently from each other, while the maximum values of $R$ and $r$ are fixed at the asymptotic region of the potential. Using such a procedure, we have obtained $R^{\min }$ 
TABLE 1: Convergence of Rovibrational Partition Function Calculations for Water Using the Hybrid LCP/QFH Method

\begin{tabular}{ccccc}
\hline & run 1 & run 2 & run 3 & all \\
& $N_{\mathrm{T}}=4 \times 10^{8}$ & $N_{\mathrm{T}}=4 \times 10^{8}$ & $N_{\mathrm{T}}=4 \times 10^{8}$ & $N_{\mathrm{T}}=1.2 \times 10^{9}$ \\
$T, \mathrm{~K}$ & $N_{\text {in }}=25992144$ & $N_{\text {in }}=25999790$ & $N_{\text {in }}=25991985$ & $N_{\text {in }}=77983919$ \\
\hline 1000 & $336.0 \pm 7.9$ & $335.9 \pm 8.0$ & $320.8 \pm 7.8$ & $330.9 \pm 4.6$ \\
2000 & $1411.3 \pm 10.0$ & $1407.1 \pm 10.0$ & $1396.4 \pm 9.9$ & $1404.9 \pm 5.8$ \\
3000 & $4158.8 \pm 16.0$ & $4156.5 \pm 15.9$ & $4160.8 \pm 15.9$ & $4158.7 \pm 9.2$ \\
4000 & $10226.3 \pm 25.6$ & $10228.8 \pm 25.6$ & $10257.6 \pm 25.6$ & $10237.5 \pm 14.8$ \\
5000 & $22070.6 \pm 39.6$ & $22083.0 \pm 40.0$ & $22143.1 \pm 39.7$ & $22098.9 \pm 22.9$ \\
6000 & $42842.8 \pm 58.7$ & $42876.3 \pm 58.7$ & $42968.5 \pm 58.9$ & $42895.9 \pm 33.9$
\end{tabular}

TABLE 2: Rovibrational Partition Function of $\mathrm{H}_{2} \mathrm{O}$ as a Function of Temperature

\begin{tabular}{|c|c|c|c|c|c|}
\hline$T, \mathrm{~K}$ & $Q_{\text {rovib }}^{\mathrm{CM} a}$ & $Q_{\text {rovib }}^{\mathrm{LCP} / \mathrm{QFH}_{b}}$ & $Q_{\text {rovib }}^{\mathrm{VT}} c$ & $Q_{\mathrm{rovib}}^{\mathrm{HVMT} d}$ & $Q_{\text {rovib }}^{\text {Irwin } e}$ \\
\hline 500 & $124743 \pm 2703$ & $56.8 \pm 3.6$ & 96.58333 & 96.4132 & \\
\hline 1000 & $3263.3 \pm 28.9$ & $330.9 \pm 4.6$ & 304.580 & 303.670 & 304.172 \\
\hline 1200 & $2417.7 \pm 16.9$ & $469.1 \pm 4.6$ & 429.315 & 427.918 & 428.560 \\
\hline 1400 & $2178.6 \pm 12.5$ & $637.2 \pm 4.7$ & 586.027 & 583.987 & 584.696 \\
\hline 1600 & $2188.9 \pm 10.5$ & $843.4 \pm 5.0$ & 781.478 & 778.608 & 779.298 \\
\hline 1800 & $2343.7 \pm 9.7$ & $1096.4 \pm 5.3$ & 1023.29 & 1019.39 & 1019.89 \\
\hline 2000 & $2607.3 \pm 9.4$ & $1404.9 \pm 5.8$ & 1320.00 & 1314.85 & 1314.87 \\
\hline 2200 & $2968.9 \pm 9.4$ & $1778.9 \pm 6.3$ & 1681.04 & 1674.49 & 1673.58 \\
\hline 2400 & $3428.6 \pm 9.7$ & $2229.0 \pm 6.9$ & 2116.92 & 2108.87 & 2106.37 \\
\hline 2600 & $3992.0 \pm 10.1$ & $2766.8 \pm 7.6$ & 2639.20 & 2629.76 & 2624.68 \\
\hline 2800 & $4668.6 \pm 10.7$ & $3405.4 \pm 8.3$ & 3260.65 & 3250.27 & 3241.08 \\
\hline 3000 & $5470.1 \pm 11.4$ & $4158.7 \pm 9.2$ & 3995.27 & 3984.98 & 3969.40 \\
\hline 3200 & $6410.6 \pm 12.2$ & $5042.3 \pm 10.1$ & 4858.42 & 4850.13 & 4824.74 \\
\hline 3400 & $7505.9 \pm 13.2$ & $6072.9 \pm 11.2$ & 5866.89 & 5863.76 & 5823.60 \\
\hline 3600 & $8773.6 \pm 14.2$ & $7269.0 \pm 12.3$ & 7038.94 & 7045.89 & 6983.93 \\
\hline 3800 & $10232.9 \pm 15.4$ & $8650.1 \pm 13.5$ & 8394.37 & 8418.59 & 8325.24 \\
\hline 4000 & $11904.7 \pm 16.6$ & $10237.5 \pm 14.8$ & 9954.54 & 9996.1 & 9868.67 \\
\hline 4200 & $13811.2 \pm 18.0$ & $12053.8 \pm 16.2$ & 11742.4 & 11834.7 & 11637.1 \\
\hline 4400 & $15975.8 \pm 19.5$ & $14122.6 \pm 17.7$ & 13782.2 & 13932.9 & 13655.3 \\
\hline 4600 & $18423.2 \pm 21.1$ & $16469.1 \pm 19.3$ & 16099.8 & 16331.0 & 15949.9 \\
\hline 4800 & $21179.0 \pm 22.8$ & $19119.0 \pm 21.0$ & 18722.1 & 19061.2 & 18549.8 \\
\hline 5000 & $24269.5 \pm 24.6$ & $22098.9 \pm 22.9$ & 21677.3 & 22157.1 & 21485.8 \\
\hline 5200 & $27721.2 \pm 26.6$ & $25435.8 \pm 24.8$ & 24994.1 & 25653.4 & 24791.4 \\
\hline 5400 & $31561.1 \pm 28.7$ & $29157.1 \pm 26.9$ & 28702.2 & 29586.0 & 28502.3 \\
\hline 5600 & $35815.9 \pm 30.9$ & $33289.8 \pm 29.1$ & 32831.2 & 33990.8 & 32656.9 \\
\hline 5800 & $40511.9 \pm 33.2$ & $37860.6 \pm 31.5$ & 37411.2 & 38904.3 & 37296.7 \\
\hline 6000 & $45674.9 \pm 35.7$ & $42895.9 \pm 33.9$ & 42471.8 & 44362.4 & 42465.8 \\
\hline
\end{tabular}

${ }^{a}$ Standard classical results from eq 2; this work. ${ }^{b} \mathrm{Hybrid} \mathrm{LCP} / \mathrm{QFH}$ results from eq 3 ; this work. ${ }^{c}$ Reference $32 .{ }^{d}$ Reference $8 .{ }^{e}$ Reference 13

$=1.24444 a_{0}, R^{\max }=10.0 a_{0}, r^{\min }=1.23090 a_{0}$, and $r^{\max }=$ $10.0 a_{0}$. For $\theta$, we have taken $\theta^{\min }=0.0 \mathrm{rad}$ and $\theta^{\max }=\pi \mathrm{rad}$.

All results from the present work have been calculated using three distinct Monte Carlo sequences of random numbers obtained from different seeds for the pseudorandom number generator. In particular, we have used the ran 2 subroutine from Numerical Recipes. ${ }^{54}$ Each sequence has been computed from a total of $4 \times 10^{8}$ sampled points, totaling $N_{T}=1.2 \times 10^{9}$. Table 1 shows some of the values calculated for the rovibrational partition function $Q_{\mathrm{rovib}}^{\mathrm{LCP} / \mathrm{QFH}}$ using the hybrid LCP/QFH method, together with the corresponding statistical uncertainties. As expected, the various calculations coincide within their statistical uncertainties. Moreover, the Monte Carlo error of the final results is smaller than $1.4 \%$ for temperatures above 1000 $\mathrm{K}$ and decreases with $T$ (e.g., for $T=6000 \mathrm{~K}$, it is only $0.08 \%$ ). Note that the Monte Carlo efficiency $\left(\epsilon=N_{\text {in }} / N_{\mathrm{T}}\right)$ of our procedure is about $6.5 \%$.

3.1. Rovibrational Partition Function. The calculated rovibrational partition functions of $\mathrm{H}_{2} \mathrm{O}$ based on the standard classical procedure $\left(Q_{\mathrm{rovib}}^{\mathrm{CM}}\right)$ and the hybrid LCP/QFH method $\left(Q_{\text {rovib }}^{\mathrm{LCP} / \mathrm{QFH}}\right)$ are reported in Table 2 . For comparison, we also give in this table the results of Vidler and Tennyson ${ }^{32}\left(Q_{\text {rovib }}^{\mathrm{VT}}\right)$, which were obtained by performing an explicit summation over experimental and theoretical rovibrational energy levels, and of Harris et al. ${ }^{8}\left(Q_{\text {rovib }}^{\mathrm{HVMT}}\right)$, which were determined from a summation over theoretical energy levels. Also included is the

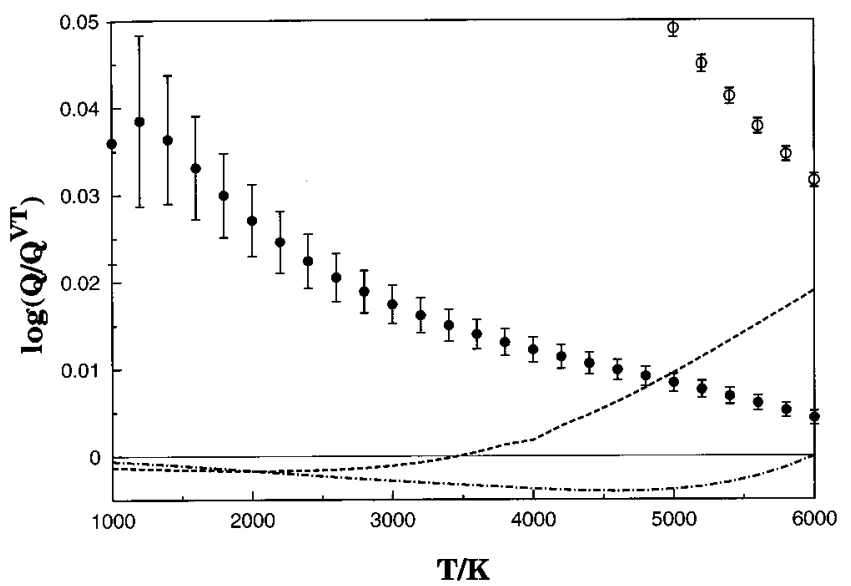

Figure 1. Logarithm of ratio of the rovibrational partition function with respect to that calculated by Vidler and Tennyson ${ }^{32}\left(Q_{\text {rovib }}^{\mathrm{VT}}\right)$ as function of temperature: $(O)$ standard classical $\left(Q_{\mathrm{rovib}}^{\mathrm{CM}}\right)$ results with error bars from eq 2 ; $(\bullet)$ hybrid LCP/QFH $\left(Q_{\text {rovib }}^{\mathrm{LCFH}}\right)$ results with error bars from eq $3 ;(---)$ Harris et al. ${ }^{8}\left(Q_{\text {rovib }}^{\mathrm{HVMT}}\right) ;(-\cdot-)$ Irwin $^{13}$ $\left(Q_{\text {rovib }}^{\text {Irwin }}\right)$.

Irwin fit $^{13}\left(Q_{\text {rovib }}^{\text {Irwin }}\right)$ to the partition function data from the JANAF thermochemical tables. ${ }^{10}$ Note that the rovibrational energy levels used in the Vidler and Tennyson calculations have been obtained from three separate sources: experiment where available,${ }^{55}$ computations from the spectroscopically determined PJT2 ${ }^{56}$ potential energy surface for levels with total angular momentum $J \leq 42$ and $E_{\mathrm{i}} \leq 30000 \mathrm{~cm}^{-1}$, and the assumption that vibrational and rotational motions can be separated for higher energies up to dissociation. In this case, the vibrational levels were computed by Mussa and Tennyson ${ }^{57}$ using an ab initio potential energy surface, ${ }^{58}$ while the rotational levels were estimated using the Padé approximant model of Polyansky. ${ }^{59}$ Regarding the calculations by Harris et al., the computed rovibrational levels were obtained from the spectroscopically determined PJT2 ${ }^{56}$ potential energy surface for $J \leq 35$ and $E_{\mathrm{i}}$ $\leq 30000 \mathrm{~cm}^{-1}$, while a procedure similar to that employed by Vidler and Tennyson was employed for higher energy levels. Assuming the results of Vidler and Tennyson as reference, we observe that our $Q_{\text {rovib }}^{\mathrm{LCP} / \mathrm{QFH}}$ values are more accurate than $Q_{\text {rovib }}^{\mathrm{CM}}$ over the whole range of temperatures, as already found in a previous study ${ }^{26}$ for diatomics. For example, at $T=2000 \mathrm{~K}$, the error relative to $Q_{\text {rovib }}^{\mathrm{VT}}$ [defined as $\Delta Q_{\text {rovib }}=\left(Q_{\text {rovib }}-\right.$ $\left.\left.Q_{\text {rovib }}^{\mathrm{VT}}\right) / Q_{\text {rovib }}^{\mathrm{VT}}\right]$ in $Q_{\text {rovib }}^{\mathrm{LCP} / \mathrm{QFH}}$ is $\sim 6.5 \%$ while that of $Q_{\text {rovib }}^{\mathrm{CM}}$ is $\sim 98 \%$. In turn, for $T=6000 \mathrm{~K}$, one observes a deviation of $1 \%$ in $Q_{\text {rovib }}^{\mathrm{LCFH}}$ and $7.5 \%$ in $Q_{\text {rovib }}^{\mathrm{CM}}$. Moreover, for temperatures above $4900 \mathrm{~K}$, the hybrid LCP/QFH approach gives results in better agreement with the Vidler and Tennyson ones than those computed by Harris et al. ${ }^{8}$ This can also be seen from Figure 1 , in which the logarithm of the ratios $Q_{\text {rovib }}^{\mathrm{CM}} / Q_{\mathrm{rovib}}^{\mathrm{VT}}, Q_{\mathrm{rovib}}^{\mathrm{LCP} / \mathrm{QFH}} /$ $Q_{\text {rovib }}^{\mathrm{VT}}, Q_{\text {rovib }}^{\mathrm{HVMT}} / Q_{\text {rovib }}^{\mathrm{VT}}$, and $Q_{\text {rovib }}^{\mathrm{Irwin}} / Q_{\text {rovib }}^{\mathrm{VT}}$ are plotted as a function of temperature. It is important to point out that the comparison between our results and previous ones (mainly those of Vidler and Tennyson ${ }^{32}$ and Harris et al. ${ }^{8}$ ) is somewhat arbitrary because of the different potential energy surfaces that have been employed for the calculations. In any case, the agreement between the hybrid LCP/QFH rovibrational partition function with previous results $\left(Q_{\mathrm{rovib}}^{\mathrm{VT}}, Q_{\mathrm{rovib}}^{\mathrm{HVMT}}\right.$, and $\left.Q_{\mathrm{rovib}}^{\mathrm{Irwin}}\right)$ is quite satisfactory at low temperatures $(T \approx 500 \mathrm{~K})$ and good at moderate and high temperatures.

The other two sets of results evaluated directly by using the Monte Carlo multidimensional integration are presented in Tables 3 and 4 (respectively, the first and second moments of 
TABLE 3: Calculated First Moment of Internal Partition Function of $\mathrm{H}_{2} \mathrm{O}$ as a Function of Temperature

\begin{tabular}{rrrr}
\hline$T, \mathrm{~K}$ & \multicolumn{1}{c}{$Q_{\text {rovib }}^{\text {'CM }}{ }^{\mathrm{CM}}$} & \multicolumn{1}{c}{$Q_{\text {rovib }}^{\mathrm{LCP} / \mathrm{QFH}}{ }_{b}$} & \multicolumn{1}{c}{$Q_{\text {rovib }}^{\mathrm{VT} c}$} \\
\hline 500 & $-1131384 \pm 28161$ & $239.4 \pm 9.5$ & 149.5118 \\
1000 & $-7279.7 \pm 108$ & $631.1 \pm 7.5$ & 553.9156 \\
1200 & $-2643.1 \pm 45.7$ & $910.4 \pm 7.1$ & 838.0108 \\
1400 & $-606.3 \pm 25.3$ & $1300.3 \pm 7.2$ & 1224.177 \\
1600 & $734.6 \pm 16.9$ & $1825.1 \pm 7.6$ & 1738.323 \\
1800 & $1911.3 \pm 13.3$ & $2512.8 \pm 8.3$ & 2409.863 \\
2000 & $3132.8 \pm 12.1$ & $3395.9 \pm 9.4$ & 3272.146 \\
2200 & $4510.6 \pm 12.2$ & $4511.8 \pm 10.7$ & 4362.889 \\
2400 & $6123.0 \pm 13.1$ & $5902.9 \pm 12.2$ & 5724.670 \\
2600 & $8036.6 \pm 14.6$ & $7617.3 \pm 13.9$ & 7405.270 \\
2800 & $10316.0 \pm 16.5$ & $9708.5 \pm 15.9$ & 9458.213 \\
3000 & $13027.6 \pm 18.7$ & $12236.5 \pm 18.2$ & 11943.2 \\
3200 & $16241.7 \pm 21.2$ & $15267.2 \pm 20.6$ & 14926.3 \\
3400 & $20033.5 \pm 23.9$ & $18873.1 \pm 23.4$ & 18480.5 \\
3600 & $24483.7 \pm 27.0$ & $23133.2 \pm 26.4$ & 22685.6 \\
3800 & $29677.9 \pm 30.3$ & $28132.0 \pm 29.7$ & 27628.2 \\
4000 & $35706.0 \pm 33.9$ & $33959.0 \pm 33.3$ & 33401.0 \\
4200 & $42661.6 \pm 37.9$ & $40708.0 \pm 37.2$ & 40102.3 \\
4400 & $50640.2 \pm 42.1$ & $48475.1 \pm 41.4$ & 47834.7 \\
4600 & $59737.6 \pm 46.7$ & $57357.1 \pm 45.9$ & 56703.6 \\
4800 & $70048.8 \pm 51.6$ & $67450.2 \pm 50.8$ & 66816.0 \\
5000 & $81665.5 \pm 56.9$ & $78847.8 \pm 56.1$ & 78278.4 \\
5200 & $94675.1 \pm 62.6$ & $91638.9 \pm 61.7$ & 91195.1 \\
5400 & $109158.5 \pm 68.6$ & $105906.4 \pm 67.7$ & 105666.5 \\
5600 & $125189.5 \pm 75.0$ & $121725.8 \pm 74.1$ & 121787.3 \\
5800 & $142833.0 \pm 81.9$ & $139164.0 \pm 80.9$ & 139645.3 \\
6000 & $162144.5 \pm 89.1$ & $158278.2 \pm 88.1$ & 159320.0
\end{tabular}

${ }^{a}$ Standard classical results from eq 7; this work. ${ }^{b}$ Hybrid LCP/QFH results from eq 9; this work. ${ }^{c}$ Reference 32.

TABLE 4: Calculated Second Moment of Internal Partition Function of $\mathrm{H}_{2} \mathrm{O}$ as a Function of Temperature

\begin{tabular}{rcrr}
\hline$T, \mathrm{~K}$ & \multicolumn{1}{c}{$Q_{\text {rovib }}^{\prime \prime \text { CM }}$} & \multicolumn{1}{c}{$Q_{\text {rovib }}^{\prime \prime \mathrm{LCP}^{\prime}}$} & \multicolumn{1}{c}{$Q_{\text {rovib }}^{\prime \prime \text { 'VT }}$} \\
\hline 500 & $10835142 \pm 300896$ & $690.9 \pm 44.9$ & 399.183 \\
1000 & $31221 \pm 469$ & $1787.7 \pm 21.7$ & 1758.363 \\
1200 & $14014 \pm 154$ & $2895.6 \pm 19.2$ & 2824.526 \\
1400 & $10237.9 \pm 69.2$ & $4471.4 \pm 19.1$ & 4343.094 \\
1600 & $10411.6 \pm 39.8$ & $6634.5 \pm 20.7$ & 6439.341 \\
1800 & $12492.6 \pm 30.2$ & $9529.0 \pm 23.6$ & 9258.888 \\
2000 & $15982.4 \pm 29.5$ & $13324.5 \pm 27.5$ & 12970.58 \\
2200 & $20828.6 \pm 32.8$ & $18217.9 \pm 32.4$ & 17769.12 \\
2400 & $27149.6 \pm 38.2$ & $24434.5 \pm 38.1$ & 23877.75 \\
2600 & $35150.5 \pm 44.7$ & $32230.9 \pm 44.8$ & 31550.88 \\
2800 & $45094.0 \pm 52.4$ & $41895.9 \pm 52.3$ & 41076.76 \\
3000 & $57288.5 \pm 61.0$ & $53752.8 \pm 60.8$ & 52779.7 \\
3200 & $72081.8 \pm 70.6$ & $68158.7 \pm 70.3$ & 67021.0 \\
3400 & $89855.7 \pm 81.3$ & $85502.7 \pm 80.9$ & 84199.0 \\
3600 & $111019.0 \pm 93.0$ & $106200.5 \pm 92.5$ & 104745.8 \\
3800 & $135998 \pm 106$ & $130686 \pm 105$ & 129121.6 \\
4000 & $165227 \pm 120$ & $159403 \pm 119$ & 157806.7 \\
4200 & $199134 \pm 135$ & $192787 \pm 135$ & 191290.4 \\
4400 & $238125 \pm 152$ & $231259 \pm 151$ & 230058.2 \\
4600 & $282578 \pm 170$ & $275206 \pm 169$ & 274578.9 \\
4800 & $332824 \pm 190$ & $324973 \pm 189$ & 325290.7 \\
5000 & $389142 \pm 211$ & $380847 \pm 209$ & 382588.9 \\
5200 & $451748 \pm 233$ & $443056 \pm 232$ & 446815.3 \\
5400 & $520790 \pm 257$ & $511758 \pm 256$ & 518249.1 \\
5600 & $596350 \pm 282$ & $587041 \pm 281$ & 597100.7 \\
5800 & $678437 \pm 310$ & $668921 \pm 308$ & 683507.8 \\
6000 & $766993 \pm 338$ & $757343 \pm 337$ & 777534.1
\end{tabular}

${ }^{a}$ Standard classical results from eq 8; this work. ${ }^{b}$ Hybrid LCP/QFH results from eq 10 ; this work. ${ }^{c}$ Ref 32 .

the $\mathrm{H}_{2} \mathrm{O}$ rovibrational partition function) and are compared with the accurate results of Vidler and Tennyson. ${ }^{32}$ It is clear that, for both cases, the hybrid LCP/QFH method shows an improved agreement with respect to the standard classical statistical mechanics results over the whole range of temperatures. In particular, the standard classical approach is seen to give values
TABLE 5: The Gibbs Enthalpy Function (in $\mathrm{J} \mathrm{K}^{-1} \mathbf{m o l}^{-1}$ ) of $\mathrm{H}_{2} \mathrm{O}$ as a Function of Temperature

\begin{tabular}{rccccc}
\hline$T, \mathrm{~K}$ & gef $^{\mathrm{CM} a}$ & gef $^{\mathrm{LCP} / \mathrm{QFH}} b$ & gef $^{\mathrm{VT} c}$ & gef $^{\mathrm{HVMT}} d$ & gef $^{\text {IANAF } e}$ \\
\hline 500 & $252.243 \pm 0.180$ & $188.270 \pm 0.526$ & 192.681 & 192.53 & 192.68 \\
1000 & $226.452 \pm 0.074$ & $207.423 \pm 0.115$ & 206.734 & 206.58 & 206.73 \\
1200 & $226.098 \pm 0.058$ & $212.465 \pm 0.081$ & 211.727 & 211.58 & 211.73 \\
1400 & $227.257 \pm 0.048$ & $217.035 \pm 0.062$ & 216.340 & 216.19 & 216.34 \\
1600 & $229.188 \pm 0.040$ & $221.258 \pm 0.049$ & 220.624 & 220.47 & 220.62 \\
1800 & $231.516 \pm 0.034$ & $225.199 \pm 0.040$ & 224.626 & 224.47 & 224.62 \\
2000 & $234.042 \pm 0.030$ & $228.901 \pm 0.034$ & 228.383 & 228.23 & 228.37 \\
2200 & $236.653 \pm 0.026$ & $232.395 \pm 0.029$ & 231.924 & 231.77 & 231.90 \\
2400 & $239.284 \pm 0.023$ & $235.703 \pm 0.026$ & 235.274 & 235.12 & 235.25 \\
2600 & $241.895 \pm 0.021$ & $238.847 \pm 0.023$ & 238.454 & 238.30 & 238.42 \\
2800 & $244.465 \pm 0.019$ & $241.842 \pm 0.020$ & 241.481 & 241.33 & 241.44 \\
3000 & $246.981 \pm 0.017$ & $244.702 \pm 0.018$ & 244.368 & 244.23 & 244.32 \\
3200 & $249.435 \pm 0.016$ & $247.439 \pm 0.017$ & 247.130 & 247.00 & 247.07 \\
3400 & $251.825 \pm 0.015$ & $250.063 \pm 0.015$ & 249.776 & 249.65 & 249.70 \\
3600 & $254.148 \pm 0.013$ & $252.584 \pm 0.014$ & 252.317 & 252.21 & 252.23 \\
3800 & $256.407 \pm 0.012$ & $255.010 \pm 0.013$ & 254.760 & 254.66 & 254.66 \\
4000 & $258.601 \pm 0.012$ & $257.346 \pm 0.012$ & 257.113 & 257.04 & 256.99 \\
4200 & $260.732 \pm 0.011$ & $259.601 \pm 0.011$ & 259.383 & 259.33 & 259.25 \\
4400 & $262.802 \pm 0.010$ & $261.777 \pm 0.010$ & 261.574 & 261.55 & 261.42 \\
4600 & $264.814 \pm 0.010$ & $263.881 \pm 0.010$ & 263.693 & 263.69 & 263.52 \\
4800 & $266.768 \pm 0.009$ & $265.917 \pm 0.009$ & 265.743 & 265.77 & 265.56 \\
5000 & $268.666 \pm 0.008$ & $267.887 \pm 0.009$ & 267.727 & 267.79 & 267.53 \\
5200 & $270.511 \pm 0.008$ & $269.796 \pm 0.008$ & 269.650 & 269.75 & 269.44 \\
5400 & $272.303 \pm 0.008$ & $271.645 \pm 0.008$ & 271.514 & 271.65 & 271.29 \\
5600 & $274.045 \pm 0.007$ & $273.437 \pm 0.007$ & 273.322 & 273.49 & 273.09 \\
5800 & $275.738 \pm 0.007$ & $275.175 \pm 0.007$ & 275.076 & 275.28 & 274.84 \\
6000 & $277.383 \pm 0.006$ & $276.861 \pm 0.007$ & 276.779 & 277.02 & 276.54
\end{tabular}

${ }^{a}$ Standard classical results; this work. ${ }^{b}$ Hybrid LCP/QFH results; this work. ${ }^{c}$ Reference 32. ${ }^{d}$ Reference 8. ${ }^{e}$ Reference 10.

of the correct magnitude only at $T \leq 1600 \mathrm{~K}$, while the hybrid LCP/QFH method gives acceptable results from $T=$ $500 \mathrm{~K}$ upward. The only exception is for the second moment of the internal partition function in which, unexpectedly, $Q_{\text {rovib }}^{\prime \mathrm{CM}}$ lies closer to $Q_{\text {rovib }}^{\prime \mathrm{VT}}$ than does $Q_{\text {rovib }}^{\prime \prime \mathrm{LCP} / \mathrm{QFH}}$ at temperatures above $5300 \mathrm{~K}$. However, as noted above, the results of Vidler and Tennyson ${ }^{32}$ employ (for high temperatures) calculated high-energy rovibrational levels and a model for the highest rotationally excited states, and hence, such a behavior can partly be attributed to the use of different potential energy surfaces.

3.2. Thermodynamic Quantities. The values reported in Tables 2-4 have been used to obtain the Gibbs enthalpy function (gef), the Helmholtz function (hcf), the entropy $(S)$, and the specific heat capacity at constant pressure $\left(C_{p}\right)$. The results, with their associated errors obtained from the standard error propagation formulas, are given in Tables 5-8, respectively. For comparison, we also give the results from Vidler and Tennyson (VT) ${ }^{32}$ and Harris et al. (HVMT), ${ }^{8}$ as well as those from the JANAF thermochemical tables. ${ }^{10}$ As expected, the hybrid LCP/QFH results are more accurate than those computed from standard classical statistical mechanics. In general, the thermodynamic quantities calculated with the hybrid LCP/QFH method are in good agreement with previous studies (i.e., deviations of about $1 \%$ ) at temperatures above $1000 \mathrm{~K}$, while those obtained using the standard classical procedure show a similar agreement only at much higher temperatures. Moreover, assuming as reference the results of Vidler and Tennyson, ${ }^{32}$ Tables 5-7 show that the hybrid LCP/QFH results look better than the HVMT and JANAF ones over some range of temperatures. For example, gef ${ }^{\mathrm{LCP} / \mathrm{QFH}}$ lies closer to gef ${ }^{\mathrm{VT}}$ than gef $^{\mathrm{HVMT}}$ and gef ${ }^{\mathrm{JANAF}}$ for $T \leq 5300 \mathrm{~K}$ and $T \leq 4800 \mathrm{~K}$, respectively. In turn, for the Helmholtz function, such a pattern is observed at temperatures above $3800 \mathrm{~K}$ when comparing to the Harris et al. ${ }^{8}$ results and at $4000 \leq T \leq 5000 \mathrm{~K}$ in relation to the $\mathrm{JANAF}^{10}$ values. For the entropy, we observe a better agreement between $S^{\mathrm{LCP} / \mathrm{QFH}}$ and $S^{\mathrm{VT}}$ for temperatures above $1500 \mathrm{~K}$ when compar- 
TABLE 6: The Helmholtz Function (in $\mathrm{J} \mathrm{mol}^{-1}$ ) of $\mathrm{H}_{2} \mathrm{O}$ as a Function of Temperature

\begin{tabular}{rrcrrr}
\hline$T, \mathrm{~K}$ & \multicolumn{1}{c}{ hcf CM $a$} & \multicolumn{1}{c}{$\mathrm{hcf}^{\mathrm{LCP} / \mathrm{QFH} b}$} & $\mathrm{hcf}^{\mathrm{VT} c}$ & $\mathrm{hcf}^{\mathrm{HVMT} d}$ & $\mathrm{hcf}^{\mathrm{IANAF} e}$ \\
\hline 500 & $-37216 \pm 121$ & $18006 \pm 1800$ & 6925 & 6925 & 6925 \\
1000 & $-7666 \pm 111$ & $26741 \pm 408$ & 26003 & 26000 & 26000 \\
1200 & $4132 \pm 112$ & $34402 \pm 340$ & 34515 & 34509 & 34506 \\
1400 & $15957 \pm 117$ & $42952 \pm 307$ & 43513 & 43504 & 43493 \\
1600 & $27819 \pm 124$ & $52141 \pm 289$ & 52946 & 52936 & 52908 \\
1800 & $39716 \pm 135$ & $61812 \pm 281$ & 62756 & 62750 & 62693 \\
2000 & $51649 \pm 149$ & $71863 \pm 276$ & 72890 & 72891 & 72790 \\
2200 & $63616 \pm 163$ & $82218 \pm 274$ & 83300 & 83317 & 83153 \\
2400 & $75619 \pm 177$ & $92829 \pm 273$ & 93946 & 93992 & 93741 \\
2600 & $87660 \pm 189$ & $103655 \pm 272$ & 104797 & 104892 & 104520 \\
2800 & $99740 \pm 200$ & $114669 \pm 272$ & 115828 & 115998 & 115464 \\
3000 & $111860 \pm 209$ & $125848 \pm 271$ & 127019 & 127302 & 126549 \\
3200 & $124022 \pm 216$ & $137172 \pm 271$ & 138353 & 138795 & 137757 \\
3400 & $136222 \pm 223$ & $148623 \pm 270$ & 149816 & 150471 & 149073 \\
3600 & $148456 \pm 227$ & $160184 \pm 269$ & 161394 & 162321 & 160485 \\
3800 & $160717 \pm 231$ & $171838 \pm 269$ & 173072 & 174334 & 171980 \\
4000 & $172992 \pm 234$ & $183562 \pm 267$ & 184883 & 186490 & 183552 \\
4200 & $185266 \pm 236$ & $195334 \pm 266$ & 196660 & 198766 & 195191 \\
4400 & $197519 \pm 238$ & $207127 \pm 264$ & 208529 & 211130 & 206892 \\
4600 & $209729 \pm 239$ & $218915 \pm 263$ & 220418 & 223545 & 218650 \\
4800 & $221870 \pm 239$ & $230668 \pm 261$ & 232301 & 235973 & 230458 \\
5000 & $233917 \pm 239$ & $242356 \pm 259$ & 244149 & 248371 & 242313 \\
5200 & $245845 \pm 239$ & $253951 \pm 257$ & 255936 & 260697 & 254215 \\
5400 & $257629 \pm 239$ & $265425 \pm 255$ & 267634 & 272911 & 266164 \\
5600 & $269247 \pm 238$ & $276753 \pm 253$ & 279218 & 284978 & 278161 \\
5800 & $280680 \pm 237$ & $287913 \pm 250$ & 290663 & 296864 & 290204 \\
6000 & $291911 \pm 236$ & $298888 \pm 248$ & 301850 & 308544 & 302295
\end{tabular}

${ }^{a}$ Standard classical results; this work. ${ }^{b}$ Hybrid LCP/QFH results; this work. ${ }^{c}$ Reference 32. ${ }^{d}$ Reference $8 .{ }^{e}$ Reference 10 .

TABLE 7: The Entropy (in $\mathrm{J} \mathrm{K}^{-1} \mathrm{~mol}^{-1}$ ) of $\mathrm{H}_{2} \mathrm{O}$ as a Function of Temperature

\begin{tabular}{rccccc}
\hline$T, \mathrm{~K}$ & $S^{\mathrm{CM} a}$ & $S^{\mathrm{LCP} / \mathrm{QFH} b}$ & $S^{\mathrm{VT} c}$ & $S^{\mathrm{HVMT} d}$ & $S^{\mathrm{JANAF} e}$ \\
\hline 500 & $177.81 \pm 0.42$ & $224.28 \pm 4.13$ & 206.53 & 206.38 & 206.53 \\
1000 & $218.79 \pm 0.18$ & $234.16 \pm 0.52$ & 232.74 & 232.58 & 232.73 \\
1200 & $229.54 \pm 0.15$ & $241.13 \pm 0.36$ & 240.49 & 240.33 & 240.48 \\
1400 & $238.66 \pm 0.13$ & $247.72 \pm 0.28$ & 247.42 & 247.26 & 247.40 \\
1600 & $246.57 \pm 0.12$ & $253.85 \pm 0.23$ & 253.71 & 253.55 & 253.69 \\
1800 & $253.58 \pm 0.11$ & $259.54 \pm 0.20$ & 259.49 & 259.33 & 259.45 \\
2000 & $259.87 \pm 0.10$ & $264.83 \pm 0.17$ & 264.83 & 264.67 & 264.76 \\
2200 & $265.57 \pm 0.10$ & $269.77 \pm 0.15$ & 269.79 & 269.64 & 269.70 \\
2400 & $270.79 \pm 0.10$ & $274.38 \pm 0.14$ & 274.42 & 274.28 & 274.31 \\
2600 & $275.61 \pm 0.09$ & $278.71 \pm 0.13$ & 278.76 & 278.64 & 278.62 \\
2800 & $280.09 \pm 0.09$ & $282.80 \pm 0.12$ & 282.85 & 282.76 & 282.68 \\
3000 & $284.27 \pm 0.09$ & $286.65 \pm 0.11$ & 286.71 & 286.66 & 286.50 \\
3200 & $288.19 \pm 0.08$ & $290.30 \pm 0.10$ & 290.36 & 290.36 & 290.12 \\
3400 & $291.89 \pm 0.08$ & $293.78 \pm 0.09$ & 293.84 & 293.90 & 293.55 \\
3600 & $295.39 \pm 0.08$ & $297.08 \pm 0.09$ & 297.15 & 297.29 & 296.81 \\
3800 & $298.70 \pm 0.07$ & $300.23 \pm 0.08$ & 300.30 & 300.54 & 299.91 \\
4000 & $301.85 \pm 0.07$ & $303.24 \pm 0.08$ & 303.32 & 303.66 & 302.88 \\
4200 & $304.84 \pm 0.07$ & $306.11 \pm 0.07$ & 306.21 & 306.65 & 305.72 \\
4400 & $307.69 \pm 0.06$ & $308.85 \pm 0.07$ & 308.97 & 309.53 & 308.44 \\
4600 & $310.41 \pm 0.06$ & $311.47 \pm 0.07$ & 311.61 & 312.28 & 311.06 \\
4800 & $312.99 \pm 0.06$ & $313.97 \pm 0.06$ & 314.14 & 314.93 & 313.57 \\
5000 & $315.45 \pm 0.06$ & $316.36 \pm 0.06$ & 316.56 & 317.46 & 315.99 \\
5200 & $317.79 \pm 0.05$ & $318.63 \pm 0.06$ & 318.87 & 319.88 & 318.32 \\
5400 & $320.01 \pm 0.05$ & $320.80 \pm 0.05$ & 321.08 & 322.18 & 320.58 \\
5600 & $322.13 \pm 0.05$ & $322.86 \pm 0.05$ & 323.18 & 324.38 & 322.76 \\
5800 & $324.13 \pm 0.05$ & $324.82 \pm 0.05$ & 325.19 & 326.46 & 324.87 \\
6000 & $326.04 \pm 0.05$ & $326.68 \pm 0.05$ & 327.10 & 328.44 & 326.92
\end{tabular}

${ }^{a}$ Standard classical results; this work. ${ }^{b}$ Hybrid LCP/QFH results; this work. ${ }^{c}$ Reference 32. ${ }^{d}$ Reference $8 .{ }^{e}$ Reference 10.

ing to $S^{\mathrm{HVMT}}$ and for temperatures over the range $2000 \leq T \leq$ $5700 \mathrm{~K}$ when considering $S^{\mathrm{JANAF}}$.

For the specific heat capacity at constant pressure, the agreement between the various results is worse than for the other thermodynamic quantities. In fact, $C_{p}$ is particularly sensitive to convergence of the partition function, because it is determined from the difference between the second and the square of the
TABLE 8: The Specific Heat Capacity at Constant Pressure (in $\mathrm{J} \mathrm{K}^{-1} \mathrm{~mol}^{-1}$ ) of $\mathrm{H}_{2} \mathrm{O}$ as a Function of Temperature

\begin{tabular}{cccccc}
\hline$T, \mathrm{~K}$ & $C_{p}^{\mathrm{CM}} a$ & $C_{p}^{\mathrm{LCP} / \mathrm{QFH}}{ }_{b}$ & $C_{p}^{\mathrm{VT}} c$ & $C_{p}^{\mathrm{HVMT}} d$ & $C_{p}^{\mathrm{JANAF} e}$ \\
\hline 1000 & $58.96 \pm 1.40$ & $35.46 \pm 2.72$ & 41.287 & 41.278 & 41.268 \\
1200 & $59.05 \pm 0.66$ & $40.79 \pm 1.94$ & 43.809 & 43.795 & 43.768 \\
1400 & $59.21 \pm 0.44$ & $44.51 \pm 1.58$ & 46.124 & 46.114 & 46.054 \\
1600 & $59.40 \pm 0.39$ & $47.26 \pm 1.37$ & 48.157 & 48.160 & 48.050 \\
1800 & $59.58 \pm 0.41$ & $49.38 \pm 1.24$ & 49.904 & 49.929 & 49.749 \\
2000 & $59.75 \pm 0.46$ & $51.07 \pm 1.15$ & 51.394 & 51.452 & 51.180 \\
2200 & $59.93 \pm 0.50$ & $52.45 \pm 1.08$ & 52.668 & 52.776 & 52.408 \\
2400 & $60.11 \pm 0.54$ & $53.62 \pm 1.03$ & 53.766 & 53.953 & 53.444 \\
2600 & $60.30 \pm 0.57$ & $54.62 \pm 0.98$ & 54.724 & 55.026 & 54.329 \\
2800 & $60.50 \pm 0.59$ & $55.50 \pm 0.93$ & 55.571 & 56.033 & 55.089 \\
3000 & $60.70 \pm 0.61$ & $56.27 \pm 0.89$ & 56.326 & 56.996 & 55.748 \\
3200 & $60.91 \pm 0.61$ & $56.95 \pm 0.85$ & 57.005 & 57.928 & 56.323 \\
3400 & $61.09 \pm 0.61$ & $57.55 \pm 0.82$ & 57.614 & 58.824 & 56.828 \\
3600 & $61.25 \pm 0.61$ & $58.05 \pm 0.79$ & 58.152 & 59.671 & 57.276 \\
3800 & $61.35 \pm 0.61$ & $58.46 \pm 0.76$ & 58.613 & 60.441 & 57.675 \\
4000 & $61.39 \pm 0.60$ & $58.76 \pm 0.73$ & 58.986 & 61.104 & 58.033 \\
4200 & $61.34 \pm 0.59$ & $58.94 \pm 0.70$ & 59.259 & 61.627 & 58.357 \\
4400 & $61.18 \pm 0.57$ & $58.98 \pm 0.67$ & 59.418 & 61.981 & 58.650 \\
4600 & $60.90 \pm 0.56$ & $58.88 \pm 0.65$ & 59.451 & 62.143 & 58.918 \\
4800 & $60.49 \pm 0.55$ & $58.63 \pm 0.62$ & 59.350 & 62.098 & 59.164 \\
5000 & $59.96 \pm 0.53$ & $58.23 \pm 0.60$ & 59.111 & 61.844 & 59.390 \\
5200 & $59.30 \pm 0.51$ & $57.69 \pm 0.57$ & 58.734 & 61.384 & 59.628 \\
5400 & $58.52 \pm 0.50$ & $57.02 \pm 0.55$ & 58.255 & 60.732 & 59.864 \\
5600 & $57.64 \pm 0.48$ & $56.24 \pm 0.53$ & 57.591 & 59.907 & 60.100 \\
5800 & $56.67 \pm 0.47$ & $55.35 \pm 0.51$ & 56.846 & 58.934 & 60.335 \\
6000 & $55.63 \pm 0.45$ & $54.38 \pm 0.49$ & 56.003 & 57.838 & 60.571
\end{tabular}

${ }^{a}$ Standard classical results; this work. ${ }^{b}$ Hybrid LCP/QFH results; this work. ${ }^{c}$ Reference $32 .{ }^{d}$ Reference $8 .{ }^{e}$ Reference 10.

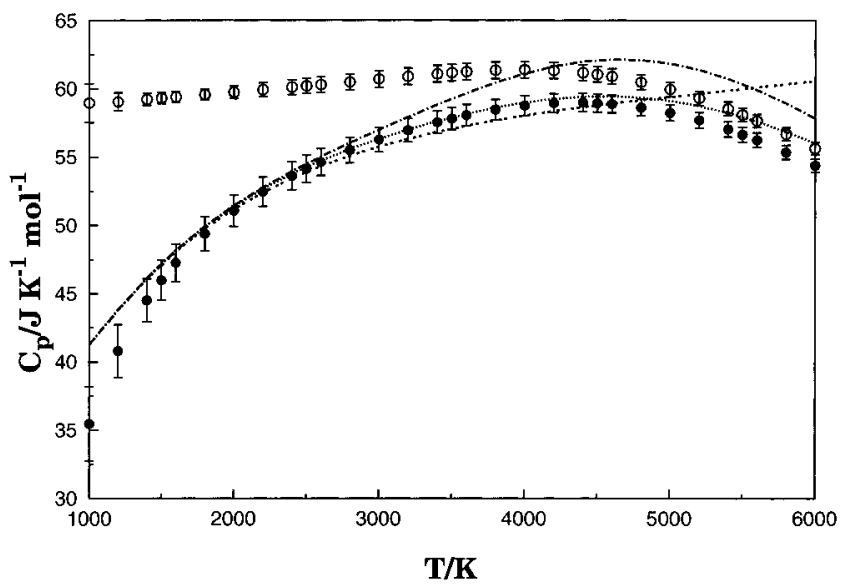

Figure 2. Specific heat at constant pressure, $C_{p}$, as function of temperature: $(\mathrm{O})$ standard classical results $\left(C_{p}^{\mathrm{CM}}\right)$ with error bars, this work; $(\bullet)$ hybrid LCP/QFH results $\left(C_{p}^{\mathrm{LCP} / \mathrm{QFH}}\right)$ with error bars, this work; $(\cdots) C_{p}^{\mathrm{VT}}$ from ref $32 ;(-\cdot-) C_{p}^{\mathrm{HVMT}}$ from ref $8 ;(--) C_{p}^{\mathrm{JANAF}}$ from ref 13 .

first moments. In particular, previous workers ${ }^{8,32}$ have called attention to the fact that it is difficult to obtain reliable results for $C_{p}$ at high temperatures. In our calculations, this can be quantified from the size of the Monte Carlo error associated with the $C_{p}$ results, which is proportionally higher than the Monte Carlo error for the other thermodynamic quantities. However, conversely to previous calculations, the uncertainties in both sets of calculations reported in the present work decrease with temperature. This is particularly clear in Figure 2, which shows $C_{p}$ as a function of temperature. In any case, $C_{p}^{\mathrm{LCP} / \mathrm{QFH}}$ follows much better the general behavior of the Vidler and Tennyson, ${ }^{32}$ Harris et al., ${ }^{8}$ and $\mathrm{JANAF}^{10}$ results than $C_{p}^{\mathrm{CM}}$, specially for $T \leq 4000 \mathrm{~K}$. Moreover, if we assume $C_{p}^{\mathrm{VT}}$ as reference, then we can claim that the hybrid LCP/QFH results are more accurate than $C_{p}^{\mathrm{HVMT}}$ at temperatures above $2400 \mathrm{~K}$ 
and $C_{p}^{\mathrm{JANAF}}$ at $T \geq 2200 \mathrm{~K}$. Finally, we comment on an interesting feature that is observed at temperatures above 4000 $\mathrm{K}$. While the JANAF specific heat value continues to increase with temperature, both our results (based on standard classical statistical mechanics and the hybrid LCP/QFH method) and those of Vidler and Tennyson ${ }^{32}$ and Harris et al. ${ }^{8}$ show a maximum at about $4500 \mathrm{~K}$ before decreasing for higher temperatures. Such a feature may be attributed to a saturation of the energy levels of water in its ground electronic state, given that the phase-space hypervolume $\mathscr{B}$ associated with a boundstate regime is finite. A similar explanation was suggested by Vidler and Tennyson ${ }^{32}$ on the basis of the fact that the number of rovibrational energy levels is finite; see also ref 60 . Such a feature is absent from the JANAF results because the results for $T \geq 4000 \mathrm{~K}$ have been obtained using a linear extrapolation.

\section{Conclusions}

Through the use of the standard classical statistical mechanics and hybrid LCP/QFH methods, calculations of the rovibrational partition function of water and related thermodynamic quantities have been reported as a function of temperature. The hybrid $\mathrm{LCP} / \mathrm{QFH}$ results are found to be rather more accurate than the standard classical ones, both for the partition function (as shown previously for diatomic systems ${ }^{26}$ ) and for thermodynamic properties (Gibbs enthalpy function, Helmholtz function, entropy, and specific heat at constant pressure). Moreover, the hybrid LCP/QFH results have been found to be in good agreement with previous calculations based on experimental or theoretical rovibrational energy levels or both ${ }^{8,32}$ and an approximate compilation ${ }^{10}$ for temperatures between 1000 and $6000 \mathrm{~K}$. In summary, our hybrid LCP/QFH method can provide accurate values of the partition function and related thermodynamic properties of polyatomic molecules described by realistic potential energy surfaces at moderate- and high-temperature regimes in which the exact sum-over-states quantum mechanical treatment is unaffordable.

Acknowledgment. The authors thank Dr. Antonio Riganelli (University of Perugia) for helpful discussions. This work has the support of Fundação para a Ciência e Tecnologia, Portugal. F.V.P. also acknowledges partial financial help through Fundação Coordenação de Aperfeiçoamento de Pessoal de Nível Superior (CAPES, Brazil).

\section{References and Notes}

(1) Bačić, Z.; Light, J. C. Аnnu. Rev. Phys. Chem. 1989, 40, 469.

(2) Carrington, T., Jr. Encyclopedia of Computational Chemistry; John Wiley \& Sons: New York, 1998; p 3157.

(3) Tennyson, J. Computational Molecular Spectroscopy; Jensen, P., Bunker, P. R., Eds.; Wiley: New York, 2000; p 305.

(4) Light, J. C.; Carrington, T., Jr. Adv. Chem. Phys. 2000, 114, 263.

(5) Prudente, F. V.; Costa, L. S.; Acioli, P. H. J. Phys. B: At., Mol. Opt. Phys. 2000, 33, R285.

(6) Neale, L.; Tennyson, J. Astrophys. J. 1995, 454, L169.

(7) Partridge, H.; Schwenke, D. W. J. Chem. Phys. 1997, 106, 4618

(8) Harris, G. J.; Viti, S.; Mussa, H. Y.; Tennyson, J. J. Chem. Phys. 1998, $109,7197$. 6325.

(9) Koput, J.; Carter, S.; Handy, N. C. J. Phys. Chem. A 1998, 102,

(10) Chase, M. W., Jr.; Davies, C. A.; Downey, J. R., Jr.; Frurip, D. J.; McDonald, R. A.; Syveraud, A. N. JANAF Thermodynamic Tables, 3rd ed; American Chemical Society and American Institute for Physics for the National Bureau of Standards: New York, 1985.
(11) Friedman, A. S.; Haar, L. J. Chem. Phys. 1954, 22, 2051.

(12) Woolley, H. W. J. Res. Natl. Bur. Stand. 1987, 92, 35.

(13) Irwin, A. W. Astron. Astrophys. Suppl. 1988, 74, 145.

(14) Martin, J. M. L.; François, J. P.; Gijbels, R. J. Chem. Phys. 1992, 96, 7633.

(15) Riganelli, A.; Wang, W.; Varandas, A. J. C. J. Phys. Chem. A 1999, 103,8303 .

(16) Topper, R. Q.; Truhlar, D. G. J. Chem. Phys. 1992, 97, 3647.

(17) Topper, R. Q. Adv. Chem. Phys. 1999, 105, 117.

(18) Mielke, S. L.; Srinivasan, J.; Truhlar, D. G. J. Chem. Phys. 2000, 112,8758 .

(19) Taubmann, G.; Witschel, W.; Shoendorff, L. J. Phys. B: At., Mol. Opt. Phys. 1999, 32, 2859.

(20) Messina, M.; Schenter, G. K.; Garrett, C. J. Chem. Phys. 1993, 98, 4120 .

(21) Pitzer, K. S.; Gwinn, W. D. J. Chem. Phys. 1942, 10, 428.

(22) Dardi, P. S.; Dahler, J. S. J. Chem. Phys. 1990, 93, 3562.

(23) Riganelli, A.; Prudente, F. V.; Varandas, A. J. C. Phys. Chem. Chem. Phys. 2000, 2, 4121.

(24) Taubmann, G.; Schmatz, S. Phys. Chem. Chem. Phys. 2001, 3, 2296.

(25) Dahler, J. S. Mol. Phys. 2001, 99 (18), 1563.

(26) Prudente, F. V.; Riganelli, A.; Varandas, A. J. C. J. Phys. Chem.

A 2001, 105, 5272.

(27) Miller, W. H. J. Chem. Phys. 1971, 55, 3146.

(28) Feynman, R. P.; Hibbs, A. R. Quantum Mechanics and Path Integrals; McGraw-Hill: New York, 1965.

(29) Urbano, A. P. A.; Prudente, F. V.; Riganelli, A.; Varandas, A. J. C. Phys. Chem. Chem. Phys. 2001, 3, 5000.

(30) Barker, J. R. J. Phys. Chem. 1987, 91, 3849.

(31) Topper, R. Q.; Zhang, Q.; Liu, Y.; Truhlar, D. G. J. Chem. Phys. 1993, 98, 4991.

(32) Vidler, M.; Tennyson, J. J. Chem. Phys. 2000, 113, 9766.

(33) McQuarrie, D. A. Statistical Mechanics; Harper and Row: New York, 1976.

(34) Miranda, E. N. Eur. J. Phys. 2001, 22, 483.

(35) Landau, L.; Lifshitz, E. Statistical Physics; Pergamon Press: New York, 1969.

(36) Riganelli, A.; Prudente, F. V.; Varandas, A. J. C. J. Phys. Chem. A 2001, 105, 9518 .

(37) Smith, F. T. Phys. Rev. 1960, 120, 1058.

(38) Koonin, S. E. Computational Physics; Addison-Wesley: Redwood, 1986.

(39) Thijssen, J. M. Computational Physics; Cambridge University Press: Cambridge, U.K., 1999.

(40) Bunker, D. L. J. Chem. Phys. 1962, 37, 393.

(41) Noid, D. W.; Koszykowski, M. L.; Tabor, M.; Markus, R. A. J. Chem. Phys. 1980, 72, 6169

(42) Doll, J. D. Chem. Phys. Lett. 1980, 72, 139

(43) Farantos, S. C.; Murrell, J. N.; Hajduk, J. C. Chem. Phys. 1982 68,109 .

(44) Bhuiyan, L. B.; Hase, W. L. J. Chem. Phys. 1983, 78, 5052.

(45) Wardlaw, D. M.; Marcus, R. A. Chem. Phys. Lett. 1984, 110, 230

(46) Wardlaw, D. M.; Marcus, R. A. J. Chem. Phys. 1985, 83, 3462.

(47) Berblinger, M.; Schlier, C. Comput. Phys. Commun. 1991, 66, 157.

(48) Berblinger, M.; Schlier, C. J. Chem. Phys. 1992, 96, 6834.

(49) Berblinger, M.; Schlier, C.; Tennyson, J.; Miller, S. J. Chem. Phys. $1992,96,6842$

(50) Varandas, A. J. C. J. Chem. Phys. 1996, 105, 3524

(51) Murrell, J. N.; Carter, S. J. Phys. Chem. 1984, 88, 4887.

(52) Polyansky, O. L.; Jensen, P.; Tennyson, J. J. Chem. Phys. 1994, $101,7651$.

(53) Varandas, A. J. C.; Voronin, A. I.; Riganelli, A.; Caridade, P. J. S. B. Chem. Phys. Lett. 1997, 278, 325.

(54) Press, W. H.; Teukolsky, S. A.; Vetterling, W. T.; Flannery, B. P. Numerical Recipes in Fortran: the Art of Scientific Computing; Cambridge University Press: New York, 1992.

(55) Tennyson, J.; Zobov, N. F.; Williamson, R.; Polyansky, O. L.; Bernath, P. F. J. Phys. Chem. Ref. Data 2001, 30, 735.

(56) Polyanski, O. L.; Jensen, P.; Tennyson, J. J. Chem. Phys. 1996 105,6490 .

(57) Mussa, H. Y.; Tennyson, J. J. Chem. Phys. 1998, 109, 10885.

(58) Ho, T.-S.; Hollebeek, T.; Rabitz, H.; Harding, L. B.; Schatz, G. C. J. Chem. Phys. 1996, 105, 10472.

(59) Polyansky, O. L. J. Mol. Spectrosc. 1985, 112, 79.

(60) Vigasin, A. A. Chem. Phys. Lett. 1998, 290, 495. 